\title{
CO Abundances in the Magellanic Clouds.
}

N. BLL and Y. P. VIALA

D.A.P.H.E., Observatoire de Paris - 92190 Meudon

I. GUIDI

Unità di Ricerca GIFCO/ONR - Firenze

(Il Nuovo Cimento C, 8, 243 (1985))

The formula which appears in the caption of fig. I (p. 246) must be

$$
N_{\mathrm{co}}=T_{\mathrm{A}} \Delta V \frac{3 K}{4 \pi^{3} \mu^{2} \nu} \simeq 2.7 \cdot 10^{15} \mathrm{~cm}^{-2}
$$

instead of

$$
N_{\mathrm{CO}}=T_{\mathrm{A}} T_{\mathrm{K}} \frac{\Delta V 3\left(K T_{\mathrm{K}}\right)^{2}}{4 \pi^{3} \mu^{2} h \nu^{2}}=5.25 \cdot 10^{15} \mathrm{~cm}^{-2}
$$

C by Societa Italiana di Fisica

Proprietà letteraria riservata

Questo periodico

i iscritto

Direttore responsabile: RENATO ANGELO RICCI

Stampato in Bologna dalla Tipografa Compositori col tipi della Tipografia Monograf

Questo fasoicolo è stato licenziato dai torchi il 30-VI-1986 